\title{
Author index to Volume 1 (2005)
}

Adinolfi, E., 219

Banachewicz, W., 249

Barańska, J., 249

Basu, S., 51, 271

Bau, C., 161

Bigonnesse, F., 193

Blaesius, R., 43

Bodor, E.T., 43

Boehm, S., 31

Boeynaems, J.-M., 17, 377

Boyer, J.L., 183

Brailoiu, E., 359

Braun, N., 259, 349

Bredetean, O., 359

Bringmann, A., 383

Brown, E.G., 183

Burnstock, G., 1, 295,

Caciagli, F., 161, 297

Camden, J.M., 337

Carrasquero, L.M.G., 153

Cazenave, J.-P., 145

Cheng, N.-L., 299

Ciccarelli, R., 161

Colgan, S.P., 229

Communi, D., 17, 377

Costanzi, S., 173

Cristalli, G., 173

Cunha, R.A., 111

Delicado, E.G., 153

DiIorio, P., 161

Di Virgilio, F., 205, 219

Douglass, J.G., 183

Duhant, X., 377

Dun, N.J., 359

Eltzschig, H.K., 229

Erb, L., 337

Erlinge, D., 59

Farret, A., 145

Ferigo, M., 369

Ferrari, D., 219

Foresta, C., 369

Freund, M., 145

Fujishita, K., 211

Gachet, C., 145

Galeazzi, C., 369
Gao, Z.-G., 241

Garcia, A., 359

Gerevich, Z., 3

Goldman, M., 377

Gonzalez, F.A., 337

Gonzalez-Iglesias, A.E., 135

Gotberg, M., 59

$\mathrm{Gu}$, J.G., 11

Hannedouche, S., 17

Harden, T.K., 43, 67

Harnek, J., 59

He, M.-L., 135

Heft, M.W., 11

Hennessey, T.M., 101

Hindley, S., 161

Idzko, M., 219

Illes, P., 3, 21, 297

Inoue, K., 95, 211

Iqbal, J., 349

Irazu, M., 83

Jacobson, K.A., 59, 241

Jiang, S., 161

Jiménez, A.I., 153

Jones, A.C., 183

Kang, Y., 329

Kirley, T.L., 51, 193, 271

Kittner, H., 21

Klepeis, V.E., 281

Klotz, K.-N., 173

Knowles, A.F., 193

Koizumi, S., 95, 211

Kong, Q., 337

Krügel, U., 21

Krzemiński, P., 249

Kukulski, F., 193

Kunapuli, S.P., 359

Lambertucci, C., 173

Latchoumanin, O., 145

Lavoie, É.G., 193

Lechner, S.G., 31

Lecka, J., 193

Lee, S.Y., 75

Léon, C., 145

Lévesque, S.A., 193

Li, B., 241
Liang, L., 299

Liao, Z., 337

Martini, C., 173

Mediero, A., 83

Meshki, J., 359

Middlemiss, P.J., 161

Miras-Portugal, M.T., 153

Müller, C.E., 349

Murphy-Piedmonte, D.M., 51

Neary, J.T., 329

Nicholas, R.A., 67, 75

Niebauer, R.T., 241

Norgauer, J., 219

O'Grady, S.M., 75

Okada, Y., 311

Olivecrona, G.K., 59

Olteanu, D., 299

Panther, E., 219

Patel, R.I., 183

Peláez, T., 83

Pelletier, J., 259

Pendergast, W., 183

Peral, A., 83

Pérez-Sen, R., 153

Petit, P., 145

Picher, M., 183

Pintor, J., 83

Pizzirani, C., 219

Pomorski, P., 249

Qi, A.-D., 67

Rathbone, M.P., 161

Redick, C.C., 183

Regenthal, R., 21

Reichenbach, A., 383

Richards Williams, C., 299

Rideout, J.L., 183

Robson, S.C., 193

Rossato, M., 369

Sabirov, R., 311

Sánchez-Nogueiro, J., 83

Schandené, L., 377

Schwiebert, E.M., 299

Searl, T.J., 389 
Sévigny, J., 193, 259

Seye, C.I., 337

Shaver, S.R., 183

Shi, Y.-F., 329

Silinsky, E.M., 389

Simonyi, A., 337

Spies, O., 21

Stojilkovic, S.S., 135

Suarez Gonzalez, N., 17, 377

Sun, G.Y., 337

Supłat, D., 249

Tomic, M., 135

Trincavelli, M.L., 173

Trinkaus-Randall, V., 281
Tsuda, M., 95

Tuluc, F., 359

Uckermann, O., 383

Uhlmann, S., 383

Vittori, S., 173

Vollmayer, P., 349

Volpini, R., 173

Vorhoff, T., 259

Waldo, G.L., 43

Wang, L., 59

Wang, M., 337
Weinger, I., 281

Weisman, G.A., 337

Weissmuller, T., 229

Welty, E.A., 299

Werstiuk, E.S., 161

Wess, J., 241

Wiedemann, P., 383

Wurm, A., 383

Yerxa, B.R., 183

Yu, S., 337

Zimmermann, H., 259, 349

Zsembery, A., 299 